\title{
A IMPLANTAÇÃO DO SISTEMA DE GERÊNCIA DE PROJETOS DO EXÉRCITO (GPEX) NO ÂMBITO DO COMANDO MILITAR DO SUL
}

\author{
THE IMPLEMENTATION OF THE ARMY PROJECT MANAGEMENT SYSTEM \\ (GPEX) UNDER THE SOUTHERN MILITARY COMMAND
}

\author{
Alexandre Martins da Fonseca \\ Especialista em Administração Pública \\ Centro Universitário do Sul de Minas - Unis-MG \\ Varginha, MG - Brasil. \\ dafonsecacav2003@gmail.com \\ Fabricio Pelloso Piurcosky \\ Doutor em Administração \\ Centro Universitário do Sul de Minas - Unis-MG \\ Varginha, MG - Brasil. \\ fabricio@unis.edu.br \\ Rodrigo Franklin Frogeri \\ Doutor em Sistemas de Informação e Gestão do Conhecimento \\ Centro Universitário do Sul de Minas - Unis-MG. \\ Varginha, MG - Brasil. \\ rodrigo.frogeri@professor.unis.edu.br
}

Resumo: Este trabalho trata da implantação do Sistema de Gerência de Projetos do Exército no âmbito do Comando Militar do Sul. Tal abordagem se justifica pela necessidade de melhoria contínua na gestão de projetos no âmbito do Exército, de forma a agregar agilidade, eficiência e novas capacidades. O objetivo deste estudo é apresentar uma pesquisa exploratória sobre os aspectos da implantação do Sistema de Gerência de Projetos do Exército (GPEx) e sua situação atual. Assim sendo, são apresentadas uma revisão conceitual sobre o tema e uma pesquisa quantitativa por meio de um questionário direcionado ao âmbito das Organizações Militares (OM) do Comando Militar do Sul (CMS) como universo de amostragem. A pesquisa demonstrou que aproximadamente 50\% das OM ainda não utilizam o Módulo Projetos e que, aproximadamente 65\% das OM, utilizam o Módulo Gestão. Foram apontados pontos fracos do sistema bem como oportunidades de melhoria, principalmente quanto à interface gráfica do GPEx e ao desenvolvimento de atividades de capacitação de operadores. Concluiu-se que, embora incipiente, a utilização do sistema GPEx pode ser considerada como relevante para as atividades das OM.

Palavras-chave: Sistemas. Softwares. Gerenciamento de projetos.

\begin{abstract}
This paper deals with the implementation of the Army Project Management System under the Southern Military Command. Such an approach is justified by the need for continuous improvement in Army project management to add agility, efficiency, and new capabilities. The objective of this study is to present an exploratory research on the aspects of the implementation of the Army Project Management System (GPEx) and its current situation. Thus, a literature review and quantitative research were conducted through a questionnaire directed to the scope of the Military Organizations (OM) of the Southern Military Command (CMS) as a sampling universe. The survey showed that approximately 50\% of OMs do not yet use the Projects Module and that approximately 65\% of OMs use the Management Module. Weaknesses of the system were pointed out, as well as opportunities for improvement, mainly regarding the GPEx graphical interface and the development of operator training activities. It was concluded that, although incipient, the use of GPEx system can be considered as relevant for the activities of the OM.
\end{abstract}

Keywords: Management. Project. Project management.

\section{$\underline{\text { Cite como }}$}

American Psychological Association (APA)

Fonseca, A. M., Piurcosky, F. P., \& Frogeri, R. F. (2021, jan./jun.). A implantação do sistema de gerência de projetos do exército (GPEX) no âmbito do comando militar do Sul. Revista Inovação, Projetos e Tecnologias - IPTEC, São Paulo, 9(1), 76-89. https://doi.org/10.5585/iptec.v9i1.18797. 


\section{Introdução}

A sociedade atual tem vivido um período de ênfase na qualidade dos serviços públicos de forma a melhor atender às expectativas e necessidades da sociedade. A Administração Pública tem buscado a melhoria na prestação destes serviços executando-os por meio de projetos. Desta forma, a qualidade dos serviços é alcançada pela execução estruturada, por pessoal especializado e precedida de planejamento. Assim, o gerenciamento de projetos é uma atividade chave na busca pela excelência no setor público (PISA \& OLIVEIRA, 2013).

Neste contexto, a implantação do Sistema de Gerência de Projetos do Exército (GPEx), como software de apoio, veio a otimizar a gestão e o acompanhamento dos projetos no âmbito da Força Terrestre. Assim sendo, este trabalho aborda a implantação e a situação atual do Sistema de Gerência de Projetos do Exército (GPEx) em contraponto à questão: Como as Organizações Militares estão utilizando o GPEx?

Tal abordagem se justifica pela necessidade de melhoria contínua na gestão de projetos no âmbito do Exército, de forma a agregar agilidade, eficiência, e novas capacidades. Da mesma maneira, cabe lembrar a importância que estudos sobre o assunto têm na contribuição para as outras instituições da administração pública que, assim como o Exército, têm a incumbência de gerenciar projetos relativos às suas áreas de atuação.

O objetivo deste estudo é apresentar uma pesquisa exploratória sobre os aspectos da implantação do Sistema de Gerência de Projetos do Exército (GPEx) e sua situação atual. Este propósito será alcançado mediante revisão bibliográfica e uma pesquisa quantitativa realizada por meio de um questionário direcionado ao âmbito das Organizações Militares (OM) do Comando Militar do Sul (CMS $)^{1}$ como universo de amostragem.

\section{Metodologia}

Quanto à finalidade, o presente trabalho visa apresentar uma pesquisa aplicada sobre o panorama geral da implantação do GPEx nas OM do CMS, bem como as possíveis oportunidades de melhoria a serem adotadas.

Conforme foi mencionado na introdução, buscou-se a resposta para a questão: como as OM do CMS estão utilizando o GPEx? Para tal, foi realizada uma pesquisa exploratória sobre

\footnotetext{
${ }^{1}$ Dentro da estrutura da Força Terrestre, o CMS é um dos oito comandos militares de área e abrange a extensão territorial dos Estados do Rio Grande do Sul, Santa Catarina e Paraná. Tem o efetivo aproximado de 50 mil militares. ou seja, 25\% do efetivo total do Exército, distribuídos em um total de 160 Organizações Militares (BRASIL, 2014). Assim sendo, o CMS se configura como amostra significativa no contexto do Exército Brasileiro.
} 
a implantação, utilização e situação do sistema, por meio de um questionário que pode ser visto no Apêndice.

Conforme Gerhardt e Silveira, o questionário é

(...) um instrumento de coleta de dados constituído por uma série ordenada de perguntas que devem ser respondidas por escrito pelo informante, sem a presença do pesquisador. Objetiva levantar opiniões, crenças, sentimentos, interesses, expectativas, situações vivenciadas. (GERHARDT \& SILVEIRA, 2009).

Assim sendo, o questionário buscou levantar dados com os seguintes teores: ano de implantação do GPEx, a existência de militares operadores específicos do sistema, a realização de capacitação para os operadores do sistema, as facilidades e dificuldades de operação do sistema, a adequação das funcionalidades do sistema, a relevância da utilização do sistema nas atividades de Gerenciamento de Projetos, a situação de implantação do Módulo de Gestão do GPEx, a adequação das funcionalidades do Módulo de Gestão do GPEx, e a relevância da utilização do sistema nas atividades de gestão.

O universo da pesquisa foi constituído pelas Organizações Militares (OM) do Comando Militar do Sul (CMS) uma vez que, dentro da estrutura do Exército, este Comando Militar de Área constitui amostra significativa pela quantidade de Organizações Militares que o integram.

Ainda de acordo com Gerhardt e Silveira (2009), a análise dos dados tem como objetivo organizar as informações de forma a possibilitar inferências a respeito do problema proposto. A análise dos dados quantitativos seguiu as seguintes etapas: estabelecimento de categorias, codificação e tabulação dos resultados, e análise estatística dos dados (GERHARDT; SILVEIRA, 2009).

Desta forma, sendo a abordagem utilizada para a pesquisa quantitativa, em um primeiro momento foram tabulados e analisados os dados obtidos como um todo, em um segundo momento, os resultados foram categorizados em quatro grupos dentro dos níveis de comando de cada OM, sendo eles: nível Comando Militar de Área e Grandes Comandos, nível Grandes Unidades, nível Unidades e nível Subunidades.

\section{Aplicação prática}

A Administração Pública tem buscado melhoria na qualidade dos serviços prestados à sociedade. A Gestão de Projetos tem como objetivo proporcionar a melhoria e a efetividade nestes serviços (PISA; OLIVEIRA, 2013).

O Exército Brasileiro, pari passu a este avanço na Administração Pública, tem desenvolvido ações que iniciaram com o Programa de Excelência Gerencial do Exército (PEG EB), evoluindo para o atual Sistema de Excelência do Exército (SE EB). Nos itens em sequência 
será abordada a atual adoção do GPEx como sistema de apoio à gestão de projetos e da qualidade, em especial nas OM do CMS.

Projeto é um esforço temporário empreendido para criar um produto, serviço ou resultado único, (PMI, 2017).

A (ABNT, 2012) apresenta a Norma NBR 21.500:2012 com diretrizes para gerenciar projetos identificando os processos tanto para fases como para um projeto completo. Destaca que deve existir uma coordenação e alinhamento entre as ações. Segundo a ótica de (KERZNER, 2010, p. 10), "um projeto pode ser definido como sendo um empreendimento com objetivo identificável, que consome recursos e opera sob pressões de prazo, custo e qualidade". Para (VARGAS, 2005), "projeto são as ações executadas, de forma coordenada, por uma organização transitória, para as quais são alocados os insumos necessários para, em um determinado prazo, alcançar um objetivo".

Assim, partindo da ideia de que a melhor forma de uma organização alcançar um objetivo é por meio de um projeto, as atividades de gerenciamento de projetos crescem de importância.

Na visão de Vargas (2005, p.7), a gestão de projetos é o conjunto de ferramentas utilizadas de forma a permitir que a empresa desenvolva habilidades, conhecimentos e capacidades individuais, destinados ao controle de eventos não repetitivos, únicos e complexos, no contexto de tempo, custo e qualidade determinados. Na ótica apresentada por Pisa e Oliveira, tem-se que

(...) a própria definição de projeto remete à ideia de planejamento e execução de tarefas de forma estruturada, por pessoal qualificado, com objetivo claro e definido, cronograma e orçamento conhecidos, além do prévio estabelecimento de controles e indicadores para avaliação dos resultados alcançados, comparados em função do previsto. Essas características fazem do gerenciamento de projetos um instrumento a serviço da governança, que prima, entre outros princípios, pela efetividade, economicidade, eficiência, transparência e prestação de contas (PISA; OLIVEIRA, 2013).

De acordo com as Normas para Elaboração, Gerenciamento e Acompanhamento de Projetos no Exército Brasileiro (NEGAPEB), gerenciar projetos é

(...) identificar os requisitos, adaptar-se às diferentes necessidades, preocupações e expectativas das partes interessadas e o balanceamento das restrições conflitantes que incluem o escopo, a qualidade, o cronograma, o orçamento, os recursos e o risco. A cultura, estilo, ambiente e estrutura organizacional influenciam a maneira como os projetos são gerenciados (BRASIL, 2013).

Desta forma, conclui-se que as atividades de gestão de projetos têm adquirido importância inconteste na redução dos riscos e no alinhamento para obtenção dos melhores resultados, com o maior controle, e melhores métodos (CARVALHO, 2007) 
Nesta direção, podem-se ressaltar alguns exemplos de órgão da Administração Pública Federal que buscam alcançar os objetivos estratégicos e a melhoria dos serviços prestados por meio de boas práticas em gestão de projetos, normalmente estruturadas em publicações oficiais. Caso do Ministério da Economia, que por meio do Governo Digital, disponibiliza a Cartilha da Metodologia de Gerenciamento de Projetos (MGP), que é utilizada no âmbito dos órgãos da Administração Pública pertencentes ao Sisp (Sistema de Administração dos Recursos de Tecnologia da Informação).

Já o então Ministério do Planejamento, Desenvolvimento e Gestão (que com reestruturação do Ministério da Economia em 2019, passou a compor uma secretaria daquele Ministério) disponibiliza, no Portal do Software Público Brasileiro, o software GP Web, que é uma ferramenta de gestão estratégica e gerenciamento de projetos

Não distante da postura destes órgãos, a Força Terrestre, como ente da Administração Pública, emprega como documento normativo as Normas para Elaboração, Gerenciamento e Acompanhamento de Projetos no Exército Brasileiro (NEGAPEB), as quais foram aprovadas em portaria pelo Estado-Maior de Exército e estão em vigor desde o ano de 2013.

Aliado a estas normas, o Exército utilizou de 2008 até 2015 o sistema SISPEG-WEB como ferramenta de TI voltada à qualidade e gestão de projetos, o qual foi desenvolvido em convênio entre a Assessoria Especial do Gabinete do Comandante do Exército e a Fundação Trompowsky (BRASIL, 2013).

Visando a substituição do SISPEG-WEB, o Sistema de Gerência de Projetos do Exército GPEx foi aprovado por meio da Portaria Nr 204 do Comandante do Exército, em março de 2015, sendo adotado como software de apoio ao gerenciamento de projetos do Exército Brasileiro, com base nas Normas para Elaboração, Gerenciamento e Acompanhamento de Projetos no Exército Brasileiro (EB20-N-08.001), (BRASIL, 2015).

Desde então, as atividades de gerência dos projetos do Exército são realizadas por meio do GPEx. O GPEx é um sistema integrado onde são lançados os dados de cada projeto e por onde é possível realizar o seu acompanhamento. O GPEx oferece ferramentas para a inserção dos dados relativos às tarefas, registros, entregas e pendências, gerando gráficos e relatórios. Outra possibilidade do sistema é a visualização das OM responsáveis por cada um dos projetos, os envolvidos, quem são os gerentes de projetos, as autoridades patrocinadoras, o segmento, o período e o objeto.

Além do módulo de suporte às atividades de gestão de projetos, o Módulo e Gestão do GPEx está em processo de implantação. Conforme o Portal SE-EB, o Exército Brasileiro, desde 2003, vem atuando na Excelência Gerencial, inicialmente com o desenvolvimento do Programa 
Excelência Gerencial do Exército (PEG), com modelo baseado no então Programa de Qualidade do Serviço Público (PQSP) (BRASIL, 2015).

Ainda de acordo com o Portal SE-EB, no ano de 2007 foi estabelecido o Sistema de Excelência no Exército Brasileiro (SE-EB), com o objetivo de integrar as informações gerenciais do Exército para auxiliar as decisões do Comandante e do Alto-Comando da Força.

Neste contexto, e bem como nas atividades de gerenciamento de projetos, o Sistema SISPEG-WEB foi utilizado como ferramenta de gestão da qualidade até o ano de 2015. Com o objetivo de atender à necessidade ocasionada pela descontinuidade da ferramenta de apoio às atividades de gestão da qualidade, em consequência do fim do SISPEG-WEB, o Comando do Exército determinou, por meio da Portaria N. ${ }^{\circ}$ 091, a adoção do Módulo de Gestão do GPEx como Software de Apoio ao sistema de excelência das OM em 2018 (BRASIL, 2018).

A figura 1 apresenta, de forma sucinta, a cronologia da implantação de sistemas para gerência de projetos e ferramentas de qualidade no âmbito do Exército.

Figura 1 - Linha do tempo dos sistemas de gerenciamento de projetos e qualidade no exército

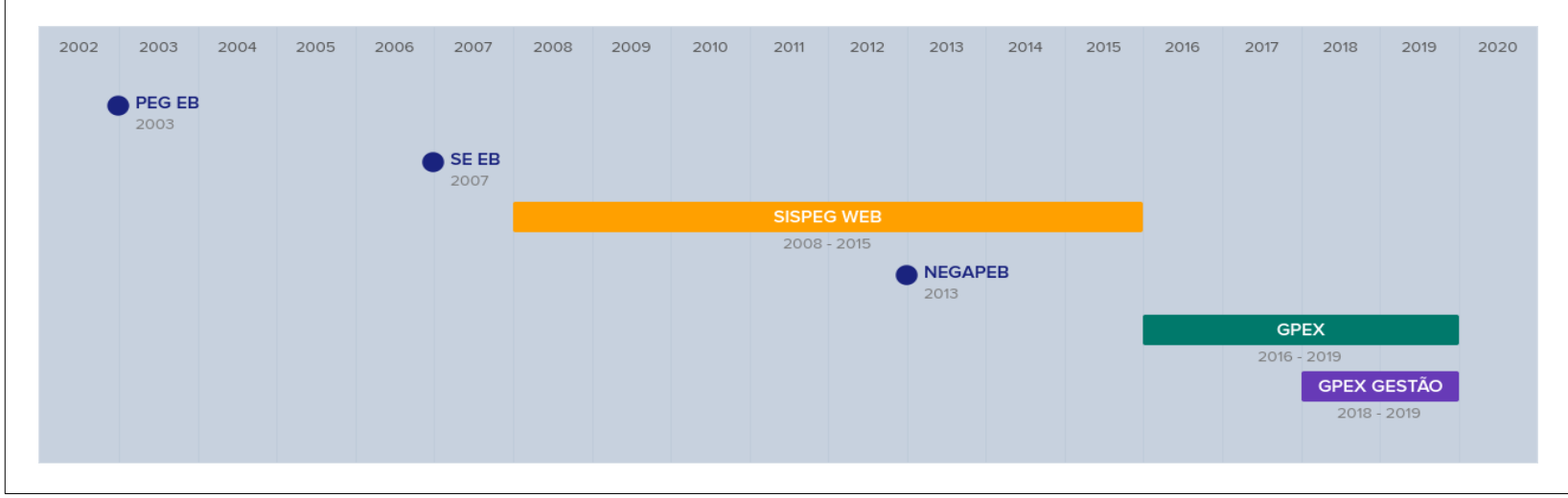

Fonte: Os autores.

A implantação deste novo módulo do GPEx tem seguido a seguinte sistemática: no ano de 2018 em caráter experimental pelo Comando Militar do Leste (CML), e em 2019 pelas demais OM (BRASIL, 2018). Assim como no GPEx Projetos, o módulo de gestão do GPEx possibilita a inserção e a edição de dados relativos à gestão da qualidade como planejamento estratégico da OM, indicadores de desempenho e planos de ação.

\section{Discussão dos resultados}

Para a realização da presente pesquisa, os questionamentos foram distribuídos, por meio de Documento Interno do Exército (DIEx), a 135 OM no âmbito do CMS. Deste total, foram obtidas 87 respostas, o que configura o percentual de 64,4\%. O gráfico 01 específica este 
universo de respostas, o qual é composto por 5,75\% de Grandes Comandos, 6,9\% de Grandes Unidades, $72,41 \%$ de Unidades e $14,94 \%$ de Subunidades.

Gráfico 1 - Composição do universo das respostas obtidas

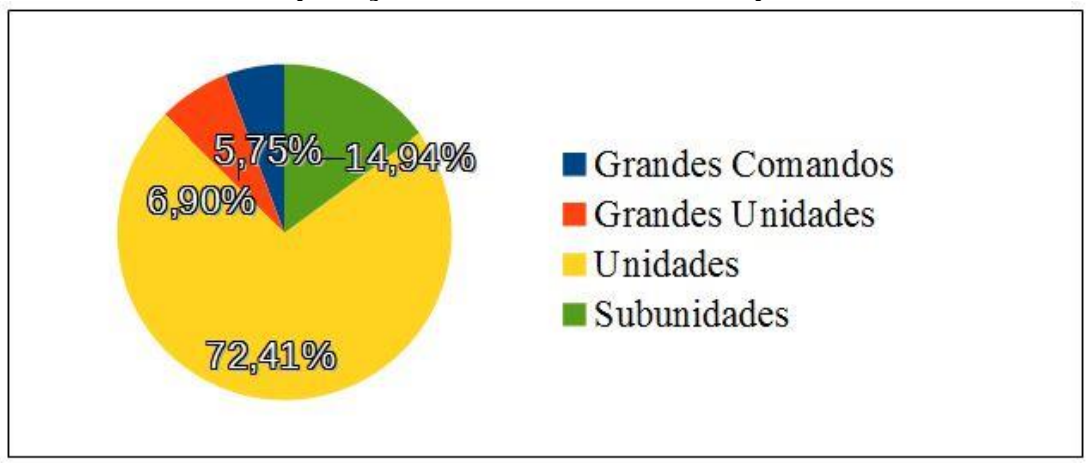

Fonte: Os autores.

Quanto ao ano de implantação do GPEx nas OM, verifica-se, conforme o gráfico 02, a minoria das OM (aproximadamente 10\%), implantaram o sistema entre os anos de 2016 e 2017, ou seja, logo após sua criação. Este percentual aumenta para 13,79\% em 2018 e, na sequência, para 26,44\% em 2019. Observa-se, no entanto, que grande parte das OM (aproximadamente $50 \%$ ) ainda não realizou a implantação.

Gráfico 2 - Ano de implantação do GPEx

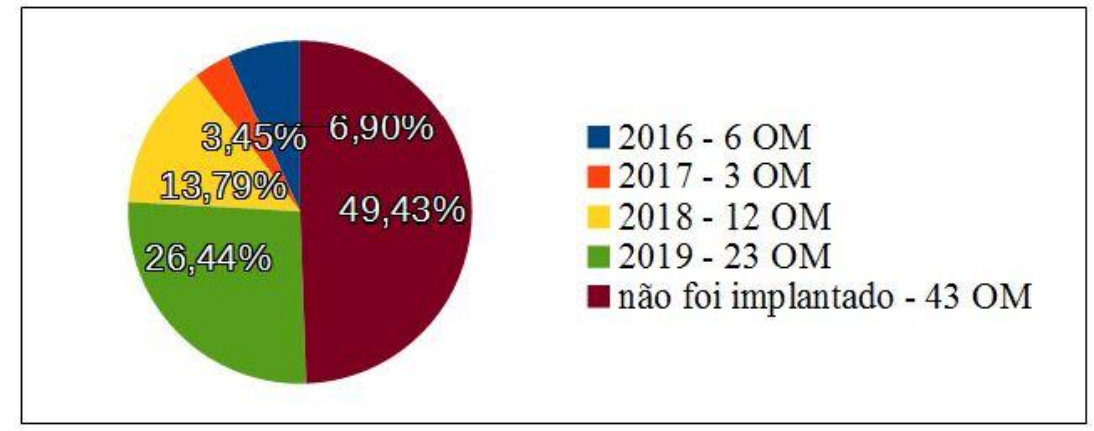

Fonte: Os autores.

Quanto à existência de militares operadores específicos do sistema, $62 \mathrm{OM}$, aproximadamente $80 \%$ das OM pesquisadas, responderam que possuem ao menos um operador do GPEx.

Quanto à facilidade de operação do GPEx por parte dos operadores, nota-se que $15 \mathrm{OM}$ (20,83\%) responderam que o sistema é de difícil operação, 46 OM $(63,89 \%)$ julgam o sistema como de dificuldade intermediária, enquanto apenas $11 \mathrm{OM}(15,28 \%)$ consideram o sistema como de fácil operação.

Percebe-se então que, embora a maioria das OM tenha ao menos um operador do sistema, grande parte considera a operação do GPEx de um nível de dificuldade considerável. 
Pode-se inferir ainda que parte desta dificuldade pode advir da ausência de atividades de capacitação, visto que $60 \%$ das $\mathrm{OM}$ pesquisadas responderam que não foi realizada nenhuma atividade/estágio de capacitação para os operadores do sistema.

Já quanto à adequação das funcionalidades do GPEx, aproximadamente 66,67\% das OM pesquisadas responderam que o sistema atende plenamente as necessidades relativas à gestão de projetos. Um quantitativo de $22,22 \%$ respondeu que o sistema atende parcialmente e $11,11 \%$ responderam que o sistema não atende.

Foram apresentados alguns pontos fracos do sistema, com destaque para a operação, propriamente dita, da maioria de suas funcionalidades. Foi levantado pelas OM que a interface gráfica é pouco intuitiva, ao mesmo tempo em que exige muitos "cliques" e a navegação em diversos menus e janelas para a execução de uma ação.

Como oportunidades de melhoria visualizadas para o sistema, as OM citaram, principalmente, a possibilidade da visualização gráfica integrada para acompanhamento das etapas dos projetos. Foi verificado, também, a importância e a necessidade de atividades de capacitação de pessoal para a operação do sistema.

Quanto ao estado de implantação do Módulo de Gestão do GPEx percebe-se que, conforme descrito no gráfico 03, apenas 20,69\% das OM já operam o sistema. Verifica-se ainda que aproximadamente $45 \%$ das $\mathrm{OM}$ estão em processo de implantação, enquanto aproximadamente $35 \%$ ainda não operam. Uma explicação para este número é a recente adoção do Módulo Gestão, a qual ocorreu no ano de 2018, em caráter experimental pelo Comando Militar do Leste (CML), e em 2019 pelas demais OM (BRASIL, 2018).

Gráfico 3 - Implantação do Módulo Gestão do GPEx

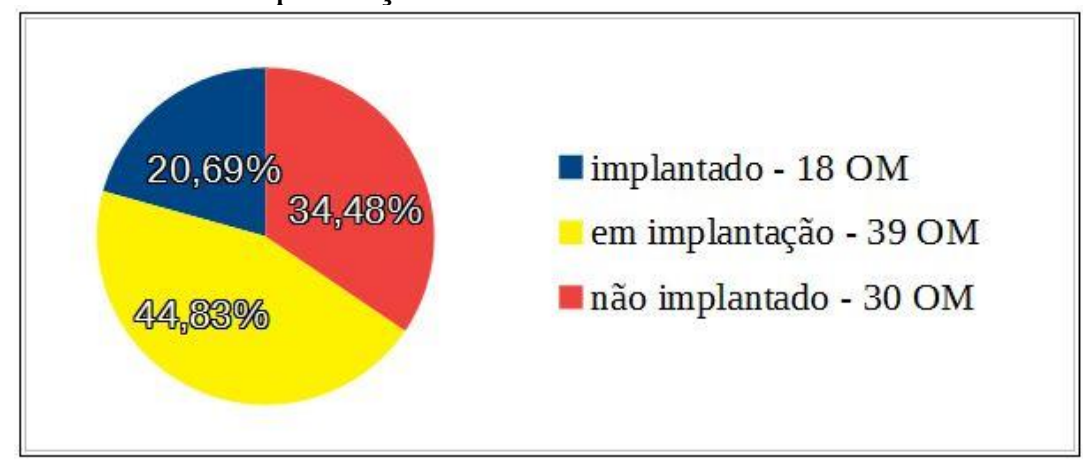

Fonte: Os autores.

Da mesma forma que no Módulo Projetos, algumas das OM que já operam, ou que estão implantando o sistema, indicaram que a interface gráfica do módulo gestão não é intuitiva 
tampouco de simples operação. A atualização de indicadores de desempenho não é automática, o que causa retrabalho.

Um ponto importante citado é o fato de o sistema não apresentar segurança para dados sensíveis. Informações relativas aos indicadores de desempenho como disponibilidade de armamento, munição, efetivos, combustível, entre outros, estão disponíveis de maneira livre a todos os operadores cadastrados.

Foram indicados alguns itens de melhoria para o módulo gestão, dentre os quais se pode destacar a compartimentação de dados, de forma a restringir o acesso às informações sensíveis; a possibilidade de correção dos dados equivocadamente lançados pelo próprio usuário, dispensando assim a solicitação de correção para o usuário do escalão superior; a simplificação da interface de operação do sistema, com a possibilidade de inserção de arquivos de formatos distintos (.pdf, .docx, .txt, etc.), a melhoria na confecção de gráficos para os indicadores de desempenho, e a possibilidade de execução de ações em "lote"; e, por fim, a realização de atividades de capacitação de pessoal para a operação do módulo gestão.

Quanto ao ganho no desenvolvimento das atividades de gestão, a utilização do sistema GPEx pode ser considerada como predominantemente relevante para as OM. Conforme observa-se no gráfico 04, o Módulo Gestão, embora ainda em fase de implantação na maior parte das OM, tem uma aceitação maior que o Módulo Projetos.

Gráfico 4 - Relevância da utilização do GPEx nas OM

\begin{tabular}{|l|c|}
\hline GPEx Projetos & GPEx Módulo Gestão \\
\hline & \\
\hline
\end{tabular}

Fonte: Os autores.

Pode-se inferir que o percentual correspondente à irrelevância do GPEx é gerado devido à pequena parcela de $\mathrm{OM}$ que desenvolve atividades peculiares com softwares de apoio específicos. Outro fator a ser considerado é que grande parte das OM do menor escalão 
considerado (subunidades, cerca de $15 \%$ do universo pesquisado) desenvolvem poucos projetos de vulto, o que limita a aplicabilidade do sistema nas atividades destas OM.

Assim, o que pode ser visto é que o fato de algumas OM ainda não possuírem as boas práticas de gestão de projetos, optam por não utilizar o sistema. Na verdade, a utilização do módulo poderia ser uma boa oportunidade para internalizar práticas com apoio de uma ferramenta.

Em muitos ambientes organizacionais, quer públicos, quer privados, esse tipo de situação existe. As recomendações para isso é buscar apoio da alta gestão para que as novas ferramentas, métodos e práticas sejam utilizadas. Este apoio de cargos hierárquicos mais altos geralmente consegue impactar os departamentos e no caso de um órgão militar isso poderia acontecer mais facilmente em vista da doutrina existente.

Dessa forma, a pesquisa consegue apontar questões importantes para melhoria da administração pública. O próprio esforço de utilização de sistemas de informação é um avanço considerável. Porém, não se pode dizer que há melhoria apenas pela elaboração e o início da utilização de uma ferramenta.

A pesquisa deixa claro que é preciso um esforço além da ferramenta, que engloba a capacitação dos usuários, ciclos de melhoria de acordo com o retorno dado pelos usuários em relação ao uso, novas funções e auditorias no uso. Ademais, seria importante ter um comitê que pudesse gerir o uso da ferramenta por criar indicadores e relacionar os mesmos às OM que utilizam o sistema àquelas que são resistentes.

\section{Conclusões}

Esta pesquisa buscou tratar da implantação do Sistema de Gerência de Projetos do Exército no âmbito do Comando Militar do Sul. Tal abordagem foi direcionada pela necessidade de melhoria contínua na gestão de projetos no âmbito do Exército.

Para tal, foi realizada uma pesquisa exploratória sobre os aspectos da implantação do Sistema de Gerência de Projetos do Exército (GPEx) e sua situação atual por meio de uma revisão bibliográfica e uma pesquisa quantitativa direcionada ao âmbito das Organizações Militares (OM) do Comando Militar do Sul (CMS) como universo de amostragem.

Quanto à implantação do GPEx, demonstrou-se que grande parte das OM (aproximadamente 50\%) ainda não realizou a implantação do sistema para o Módulo Projetos. Demonstrou-se ainda que, aproximadamente 65\% das OM pesquisadas já utilizam o Módulo 
Gestão. Conclui-se desta forma que o sistema ainda é bastante incipiente no universo pesquisado.

O estudo evidenciou alguns pontos fracos do sistema, dentre os quais se pode destacar a dificuldade na operação da maioria de suas funcionalidades, ocasionado pela interface gráfica que é pouco intuitiva. Outro aspecto importante levantado é o fato de o sistema não apresentar segurança para dados sensíveis.

Em contraponto aos pontos fracos averiguados, foram levantadas oportunidades de melhoria como o aprimoramento da interface gráfica do sistema e o desenvolvimento de atividades de treinamento para os operadores. Ainda, para o Módulo Gestão, foi verificada a necessidade de um sistema de segurança para as informações sensíveis, e a possibilidade de inserção de arquivos de formato pdf, docx, txt, etc.

Pode-se afirmar que, embora incipiente, a utilização do sistema GPEx pode ser considerada como predominantemente relevante para as OM. Verificou-se ainda que o Módulo Gestão, embora ainda em fase de implantação na maior parte das OM, tem uma aceitação maior que o Módulo Projetos, o que denota que a prática de projetos precisa ser mais bem inserida ou internalizada. Somente existir a ferramenta em si, não é garantia de que ela será utilizada. É necessário antes disso, capacitar quer para utilização da ferramenta, quer na própria área de gerenciamento, que no caso, é a área de projetos.

Atento à necessidade de melhoria contínua na gestão de projetos no âmbito do Exército e à importância da contribuição para as atividades de outras instituições da administração pública, tem-se que este assunto requer a continuidade de pesquisa. Com a adoção das inovações e melhorias sugeridas, novos resultados tendem a ser obtidos, ao mesmo tempo em que a implantação e a utilização do sistema tornam-se mais consistentes.

Essa pesquisa apresenta, portanto, uma contribuição relevante para organizações que querem trabalhar com projetos. Ela aponta que não basta ter a ferramenta ou sistema para isso. Mais uma vez, assim como outros estudos já apontaram, é preciso envolver o usuário, capacitálo e, posteriormente, criar ciclos de melhoria contínua para que a ferramenta consiga corresponder às necessidades dos usuários.

O fato de o módulo de gestão ter mais impacto de uso em um primeiro momento somente corrobora com isso. Em muitos ambientes, a gestão de projetos somente vai ter sentido e uso, se houver uma preocupação em fazer com que ela gere resultados e, para isso, desenvolver uma maneira de gerir este momento e internalizar a importância de projetos, com apoio maciço e claro da alta gestão da organização. 
A pesquisa foi limitada ao Comando Militar do Sul e seria interessante em um próximo momento verificar se a realidade desde ambiente é também retratada em outros Comandos. Como estudo futuro, seria relevante ampliar a pesquisa em torno dos efeitos do uso das ferramentas utilizadas. Verificar se houve substituição de alguma delas e como contribuíram para melhorar a gestão e os resultados.

\section{Referências}

ABNT. (2012). NBR ISO 21500:2012. Orientações para o Gerenciamento de Projetos: elaboração. Rio de Janeiro: Associação Brasileira de Normas Técnicas.

Brasil. (29 de 08 de 2013). Portaria $N^{\circ}$ 176-EME, de 29 de agosto de 2013. (C. d. Exército, Ed.) Acesso em 2019, disponível em EPEX.

Brasil. (03 de 09 de 2014). Comando Militar do Sul: Elite do combate convencional. Histórico. Acesso em 2019, disponível em Comando Militar do Sul: http://www.cms.eb.mil.br/index.php/home-2/historico

Brasil. (17 de 03 de 2015). Portaria $N^{o}$ 204-Cmt Ex, de 17 de março de 2015. Fonte: SGEX: http://www.sgex.eb.mil.br/sistemas/be/copiar.php?codarquivo=1345\&act=bre

Brasil. (22 de 05 de 2018). Portaria $N^{\circ}$ 091-EME, de 22 de maio de 2018. Fonte: Exército Brasileiro: http://www.portalse.eb.mil.br/images/Legislacao/Port091-EME_gpex.pdf

Carvalho, G. S. (05 de 2007). Passo a Passo do Gerenciamento de Projetos: Gestão \& Tecnologia de Projetos. . Gestão e Tecnologia de Projetos, p. 14.

Gerhardt, T. E., \& Silveira, D. (2009). Métodos de Pesquisa. Porto Alegre: Editora da UFRGS.

Kerzner, H. (2010). Gestão de Projetos: As Melhores Práticas. . Porto Alegre: Bookman.

Pisa, B., \& Oliveira, A. G. (2013). Gestão de Projetos na Administração Pública : Um Instrumento para o Planejamento e Desenvolvimento. 1o Seminário Nacional de Planejamento e Desenvolvimento. Curitiba.

PMI. (2017). Um Guia do conhecimento em gerenciamento de projetos. Guia PMBOK. Pensilvânia: Project Management Institute.

Vargas, R. V. (2005). Gerenciamento de Projetos: Estabelecendo Diferenciais Competitivos. . Rio de Janeiro: Brasport. 


\section{Apêndice}

\section{Instrumento de coleta de dados}

\section{GPEX - MÓDULO PROJETOS}

1. Quanto à implantação do GPEx em sua Seção, esta ocorreu em:

( ) em 2016

( ) em 2017

( ) em 2018

( ) em 2019

( ) ainda não foi implantado

2. Existem militares operadores específicos do sistema na seção?

( ) sim, apenas um

( ) sim, mais de um militar

( ) não, o sistema é operado por demanda por qualquer militar da seção

3. Foi realizada atividade/estágio de capacitação para os operadores do sistema?
( ) $\operatorname{sim}$
( ) não

4. Quanto à operação do GPEx, o sistema pode ser considerado:

( ) de fácil operação

( ) de dificuldade intermediária para operação

( ) de difícil operação

5. Quanto às funcionalidades relativas às atividades de Gerenciamento de Projetos abaixo citadas, a utilização do GPEx:
( ) atende plenamente
( ) atende parcialmente
( ) não atende

6. No caso do Módulo de Gestão do GPEx atender parcialmente, cite as oportunidades de melhoria visualizadas para o sistema:

7. Quanto ao ganho no desenvolvimento das atividades de Gerenciamento de Projetos de sua OM, a utilização do GPEx pode ser considerada como:
( ) relevante
( ) parcialmente relevante
( ) não relevante para as atividades da $\mathrm{OM}$

\section{GPEX - MÓDULO GESTÃO}

8. Quanto ao Módulo de Gestão do GPEx em sua OM encontra-se:
( ) implantado
( ) em implantação
( ) ainda não é utilizado 
9. Quanto às funcionalidades relativas ao Módulo de Gestão, caso o mesmo já esteja implantado ou em implantação, o GPEx:

( ) atende plenamente

( ) atende parcialmente

( ) não atende

10. No caso do Módulo de Gestão do GPEx atender parcialmente, cite as oportunidades de melhoria visualizadas para o sistema:

11. Quanto ao ganho no desenvolvimento das atividades de gestão em sua OM, a utilização do GPEx pode ser considerada como:

( ) relevante

( ) parcialmente relevante

( ) não relevante para as atividades da $\mathrm{OM}$ 